\title{
Enhanced Chelate Cooperativity in Polar Solvents
}

\author{
Stefan Henkel, ${ }^{\circledR}$ Maria Cristina Misuraca, ${ }^{\circledR}$ Yudi Ding, Maxime Guitet, and Christopher A. Hunter*이 \\ Department of Chemistry, University of Cambridge, Lensfield Road, Cambridge CB2 1EW, U.K.
}

\section{Supporting Information}

ABSTRACT: High-throughput UV-vis titrations in combination with chemical double-mutant cycles (DMCs) have been used to study the competition of a polar solvent for formation of intramolecular H-bonds. Twenty-four different zinc porphyrin-pyridine complexes were investigated in mixtures of toluene and phenol. DMCs were used to determine effective molarities (EM) for the formation of intramolecular phenol-amide H-bonds as a function of solvent composition. The values of EM increase by an order of magnitude with increasing concentrations of the more polar solvent, phenol. Phenol solvates the amide groups on the ligands strongly, increasing the steric bulk and destabilizing the complexes. These adverse steric interactions are removed when intramolecular H-bonds are formed and therefore provide an increased driving force for formation of cooperative interactions. The result is that the effects of competitive interactions with polar solvents that reduce binding affinity are attenuated to a significant extent by a
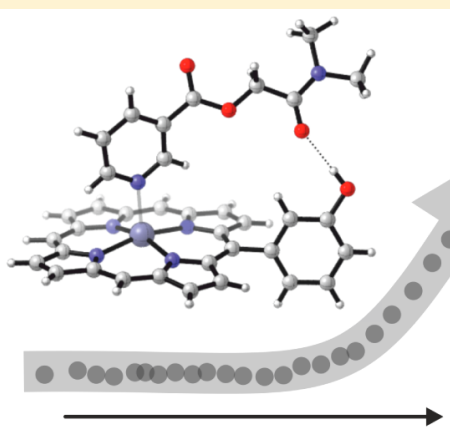

[polar solvent]

pubs.acs.org/JACS corresponding increase in EM in multivalent complexes.

\section{INTRODUCTION}

Systematic studies of the mechanisms governing molecular recognition events are fundamental to understanding and controlling biological processes and the advancement of material science and nanotechnology. Almost all systems of interest in these fields involve multiple cooperative interactions between macromolecular surfaces, and many factors can contribute to the thermodynamic properties of these interfaces: electrostatic interactions, H-bonds, aromatic interactions, and desolvation. $^{1-15}$

We have been investigating chelate cooperativity in terms of effective molarity $(\mathrm{EM})^{16-20}$ and the relationship of this parameter with the number and nature of the intermolecular interactions and the geometric complementarity and conformational flexibility of the binding partners. ${ }^{21-33}$ These experiments show that the thermodynamic properties of multivalent systems can be described as the sum of contributions from individual interaction sites. The value of EM for an intramolecular interaction does not depend on the intrinsic strength of the noncovalent interaction but is a function of the supramolecular architecture, specifically geometric complementarity. ${ }^{27}$ Although preorganization does affect EM values, the influence of conformational flexibility on the overall stability of supramolecular complexes is modest. Flexible recognition interfaces can adapt, leading to surprisingly stable complexes even when complementarity appears poor. The next step to a better understanding of cooperativity in multivalent complexes is to investigate how chelate cooperativity is affected by the solvent environment, specifically the impact of preferential solvation in solvent mixtures.

We have previously shown that molecular recognition-based probes provide insight into the thermodynamic properties of the solvation shell at individual binding sites on the surface of a molecule. ${ }^{34,35}$ This approach allows the interplay between solvent-solvent and solvent-solute interactions to be quantified. When solvent mixtures are involved, molecular recognition probes are particularly valuable in focusing measurements on solvation equilibria at specific sites on the solute. The association constant $(K)$ for formation of a 1:1 complex between a H-bond donor (D) and acceptor (A) in a mixture of a nonpolar solvent (S1) and a polar solvent (S2) was found to obey eq 1 for solvents that are not strongly selfassociated. $^{36}$

$$
K=\frac{K_{\mathrm{S} 1}}{\left(1+K_{\mathrm{D}}[\mathrm{S} 2]\right)\left(1+K_{\mathrm{A}}[\mathrm{S} 2]\right)}
$$

Here, $K_{\mathrm{S} 1}$ is the D.A association constant in $\mathrm{S} 1$ and $K_{\mathrm{D}}$ and $K_{\mathrm{A}}$ are the D.S2 and A.S2 association constants in S1.

Figure 1 illustrates eq 1 graphically: the binding constant for the D.A complex is independent of the concentration of S2 when the concentration of the polar solvent is low. However, when the concentration of S2 is increased, the polar solvent starts competing with S1 for solvation of the solutes. At this point, preferential solvation by S2 competes with the interaction of $\mathrm{A}$ with $\mathrm{D}$ leading to a less stable complex at a higher concentration of S2. A linear dependence of $\log K$ on $\log [\mathrm{S} 2]$ (slope $\approx-1$ ) was observed for solvent mixtures where $\mathrm{S} 2$ contained a good $\mathrm{H}$-bond acceptor and no strong H-bond donor sites (e.g., di-n-octyl ether) or S2 contained a H-bond donor site and no strong acceptors (e.g., 1,1,2,2-tetrachloroethane). ${ }^{36}$ When S2 contained both H-bond donor and acceptor sites, a greater dependence on $\log [\mathrm{S} 2]$ was observed. For example, the slope was -2 when $S 2$ was an alcohol. ${ }^{37}$ The

Received: February 20, 2017

Published: May 3, 2017 


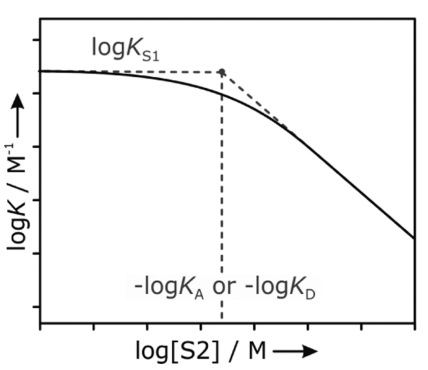

Figure 1. Association constant $(\log K)$ for formation of an intermolecular H-bond between a donor (D) and an acceptor (A) in mixtures of a nonpolar solvent (S1) and a more polar solvent (S2) as a function of $S 2$ concentration $(\log [\mathrm{S} 2])$. The horizontal dashed line corresponds to the association constant of the D.A complex in S1, and the vertical dashed line corresponds to the association constant of S2 with one of the solutes in S1.

slope of the plot of $\log K$ versus $\log [\mathrm{S} 2]$ effectively quantifies the number of S2-solute interactions that compete with formation of the D.A complex. In multivalent association, we might therefore expect that the slope of this plot could be used to measure the number of intermolecular interactions involved in binding, in effect a H-bond inventory.

\section{APPROACH}

The porphyrin-ligand system depicted in Figure 2 has been exploited for the quantitative analysis of chelate coopera-

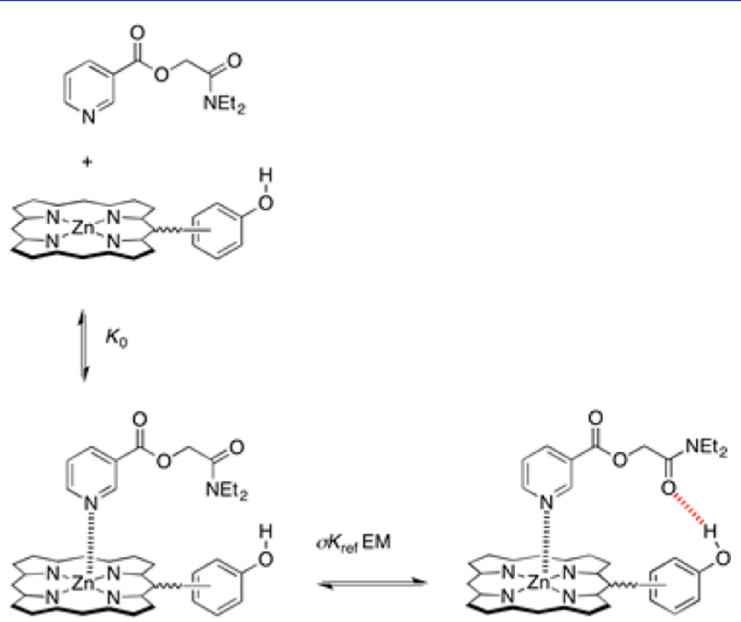

Figure 2. Stepwise equilibria in the formation of a complex between a zinc porphyrin and a pyridine ligand equipped with $\mathrm{H}$-bonding sites. $K_{0}$ is the association constant for the zinc-pyridine interaction, $K_{\text {ref }}$ is the association constant for formation of an intermolecular phenolamide H-bond, EM is the effective molarity associated with the formation of the intramolecular phenol-amide $\mathrm{H}$-bond illustrated, and $\sigma$ is a statistical factor accounting for the degeneracies of the complexes.

tivity. ${ }^{18,22-33}$ The zinc-nitrogen interaction between the porphyrin and the pyridine ligand provides a strong coordination bond that allows investigation of formation of weaker peripheral H-bonds. The ability of the system to form intramolecular $\mathrm{H}$-bonding interactions can be quantified in terms of effective molarity (EM). Strictly speaking, the H-bond in Figure 2 is an intermolecular interaction, but we will use the term intramolecular because if we consider stepwise equilibria where the zinc-nitrogen interaction is formed first the formation of the $\mathrm{H}$-bond is intramolecular.
The chemical double-mutant cycle (DMC) shown in Figure 3 can be used to determine the value of EM in such systems.

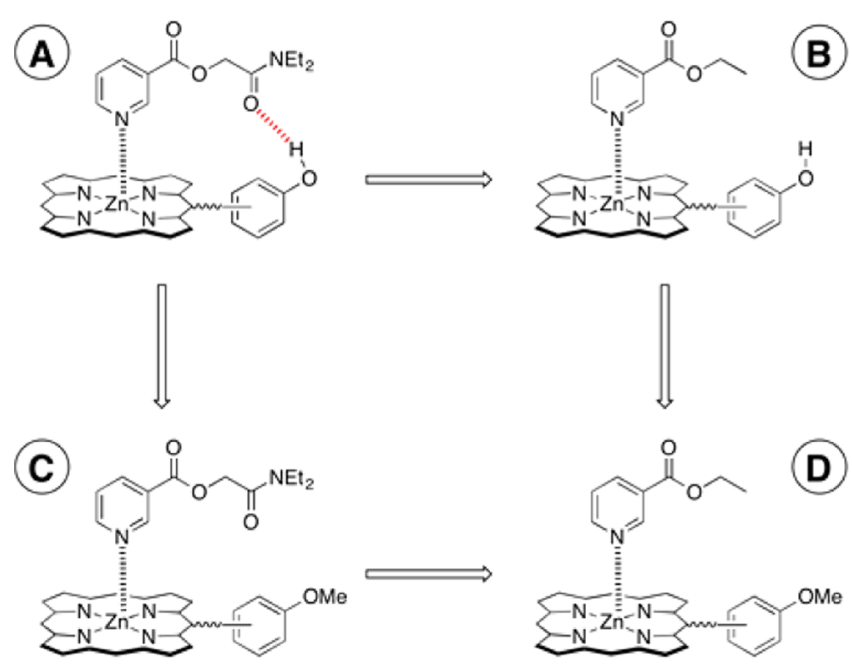

Figure 3. Chemical double mutant cycle used to determine the EM for formation of an intramolecular amide-phenol H-bond.

The DMC was introduced as an analytical tool to quantify individual contributions to the overall stability of supramolecular complexes. ${ }^{28,38,39}$ The free energy contribution due to the intramolecular H-bond in complex A can be determined using eq 2. An alternative expression (eq 3) can be used to describe this free energy contribution in terms of an apparent association constant for formation of intramolecular $\mathrm{H}$-bond in complex A, $K_{\text {intra. }}$. For porphyrin-ligand complexes that can form only one intramolecular H-bond, $K_{\text {intra }}$ can be directly related to the value of EM (eq 4). For porphyrin-ligand complexes that can form two or more $\mathrm{H}$-bonds, $K_{\text {intra }}$ represents the overall equilibrium constant for formation of singly and multiply H-bonded states

$$
\begin{aligned}
& \Delta \Delta G^{\circ}=\Delta G_{\mathrm{A}}{ }^{\circ}-\Delta G_{\mathrm{B}}{ }^{\circ}-\Delta G_{\mathrm{C}}{ }^{\circ}+\Delta G_{\mathrm{D}}{ }^{\circ} \\
& K_{\text {intra }}=e^{-\Delta \Delta G^{\circ} / R T}-1 \\
& K_{\text {intra }}=\sigma K_{\text {ref }} \mathrm{EM}
\end{aligned}
$$

where $\sigma$ is a statistical factor accounting for the degeneracies of the complexes.

Here, we describe binding studies of porphyrin-ligand complexes carried out in mixtures of two solvents, toluene (S1) and phenol (S2). The impact of the polar solvent competing for the intramolecular amide-phenol H-bonds was evaluated through application of DMCs to determine the dependence of the intramolecular equilibrium constant $K_{\text {intra }}$ on the concentration of S2.

The porphyrins and pyridine ligands used are shown in Figure 4. Synthesis of these compounds has been reported in previous publications. ${ }^{24,28}$ Porphyrins P1a-P4a are equipped with phenol moieties, which are able to interact with the $\mathrm{H}$ bond acceptors on the ligand arms. Porphyrins P1b-P4b have methoxy groups instead and cannot form H-bonds with the ligands. Ligands L2e and L3e have either one or two arms bearing amide $\mathrm{H}$-bond acceptor sites that can interact with the porphyrin phenol groups. It was shown previously that porphyrins P1a, P2a, and P3a all form H-bonds with the amide groups of L2e and L3e, but in P4a the donor and acceptor sites are too far apart and no H-bonds are observed. ${ }^{24}$ 


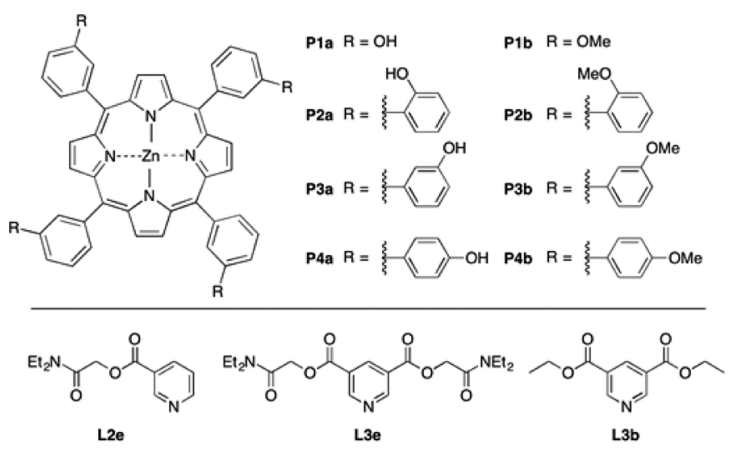

Figure 4. Porphyrins and ligands used in binding studies.

The ester carbonyl groups of L2e and L3e do not form $\mathrm{H}$ bonds in any of the complexes because they are too far from the peripheral phenol H-bond donors. Similarly, ligand L $\mathbf{3 b}$ does not form H-bonds with any of the porphyrins, so this ligand was used as the non-H-bonding control ligand in complexes $\mathrm{B}$ and $\mathrm{D}$ of the DMC.

\section{RESULTS AND DISCUSSION}

Binding of the pyridine ligands to each of the eight porphyrins was studied by UV-vis titration experiments. A shift of about $10 \mathrm{~nm}$ in the absorption maximum of the Soret band $(425 \mathrm{~nm})$ is indicative of binding of the pyridine nitrogen to the zinc of the porphyrin, and this change can be used to determine the association constant for the complex. Due to the large number of association constants measured for each porphyrin-ligand system, automated protocols were developed using a UV-vis plate reader equipped with internal syringes that were used to vary the solvent composition. The experimental data were fit to 1:1 binding isotherms using purpose-written software, which allowed analysis of 18 titration data sets in a single experimental run.

Figure 5 shows the association constants for formation of the porphyrin-ligand complexes plotted as a function of S2 concentration (see the SI for tabulated data). As expected, at low concentrations of $\mathrm{S} 2$, the stabilities of the complexes are not perturbed by the presence of the second solvent. For P4, complexes A-D all have similar stabilities, which confirms that there are no $\mathrm{H}$-bonding interactions in this system at any concentration of S2. For porphyrins P1-P3, complex A is significantly more stable than complexes B, C, and D. This observation is consistent with the presence of strong intramolecular amide-phenol H-bonds in these systems.

At higher concentrations of S2, the polar solvent starts to compete with the intramolecular phenol-amide H-bond, leading to a drop in the stability of complex A when [S2] is greater than $10 \mathrm{mM}$ for P1-P3. The concentration at which S2 begins to preferentially solvate the amide groups of the free ligand is given by the association constant for formation of an intermolecular phenol-amide $\mathrm{H}$-bond, $K_{\text {ref }}=86 \mathrm{M}^{-1}$, which was measured previously using the $p$-cresol $\cdot N, N$-diethylacetamide complex in toluene (Figure 6a). ${ }^{24}$ When $K_{\text {ref }}[\mathrm{S} 2]<1$, the amide groups of the free ligand are mainly solvated by $\mathrm{S} 1$, but when $K_{\text {ref }}[\mathrm{S} 2]>1$, they are mainly solvated by S2.

At even higher concentrations of $\mathrm{S} 2$, the association constants for complexes B, C, and D also start to decrease (Figure 5), which suggests that phenol competes for the zincnitrogen coordination bond. The decrease in log $K$ observed for complex A therefore comprises two different contributions because phenol competes for both the intramolecular $\mathrm{H}$-bond and the zinc-nitrogen interaction (Figure $6 \mathrm{~b}$ ). However, the DMC is specifically designed so that any effects due to changes in the strength of the zinc-nitrogen interaction will cancel out.

The data in Figure 5 were used in eqs 2 and 3 to determine $K_{\text {intra }}$ and the results are shown in Figure 7 . The values obtained for the ligand with two $\mathrm{H}$-bonding sites L3e are roughly twice the values found for the one-armed ligand L2e, confirming the additivity of the free energy contributions from multiple interactions in these systems.

For the P4a complexes where no H-bonds are formed, $K_{\text {intra }}$ is close to zero and constant over the entire range of phenol concentrations (not shown in Figure 7). For the P1a-P3a complexes, the $\log K_{\text {intra }}$ values are constant for phenol

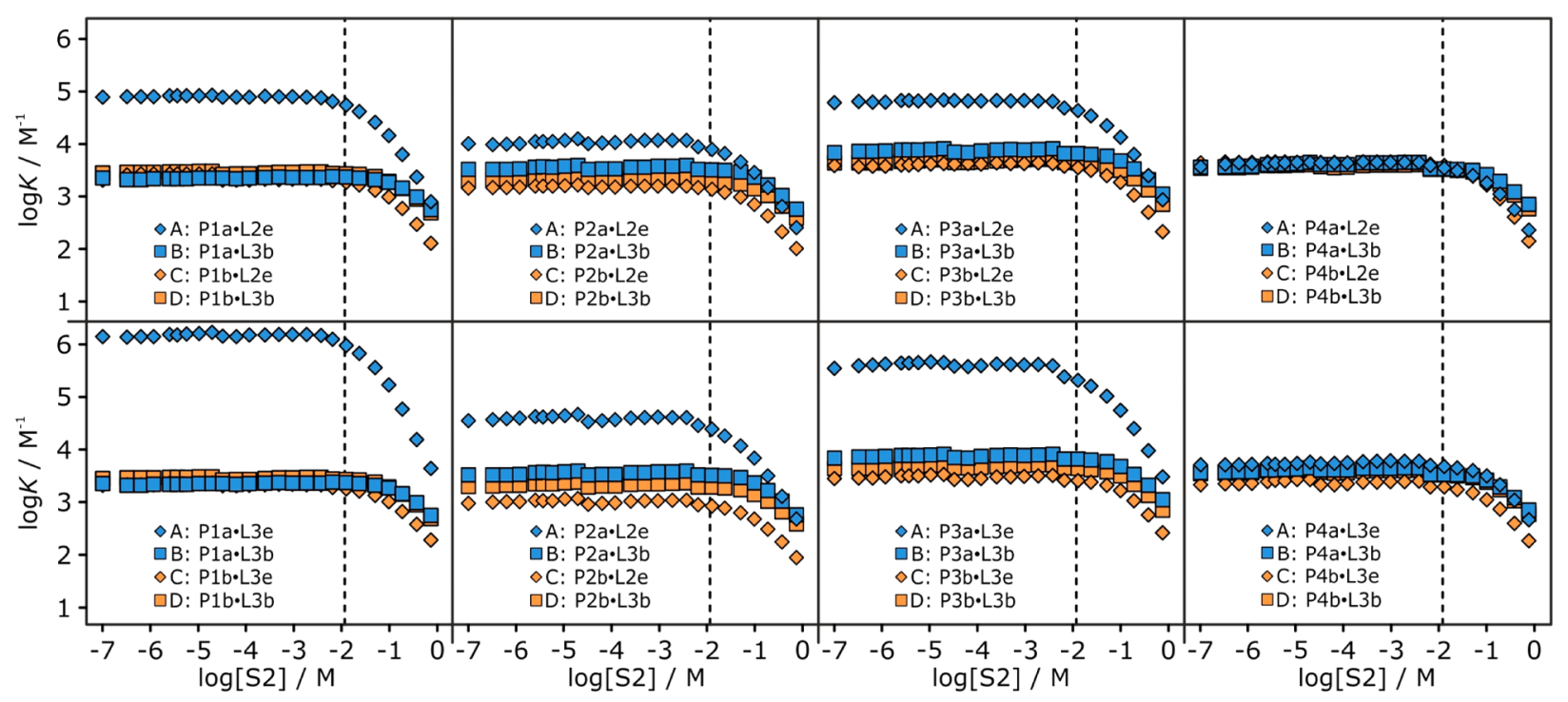

Figure 5. Association constants $(\log K)$ for formation of 1:1 porphyrin-ligand complexes in toluene-phenol mixtures plotted as a function of the concentration of phenol $(\log [\mathrm{S} 2])$. For each porphyrin-ligand combination, data for the four complexes used to construct the DMC in Figure 3 are plotted together; i.e., A corresponds to the Pa.Le complex, B to $\mathbf{P a} \cdot \mathbf{L b}, \mathbf{C}$ to $\mathbf{P b} \cdot \mathbf{L e}$, and $\mathrm{D}$ to $\mathbf{P b} \cdot \mathbf{L b}$. The vertical dashed line corresponds to $-\mathrm{log}$ $K_{\text {ref }}$ the association constant for formation of a phenol-amide $\mathrm{H}$-bond in toluene. 


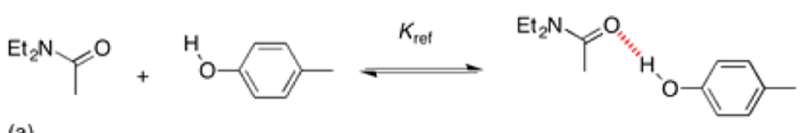

(a)
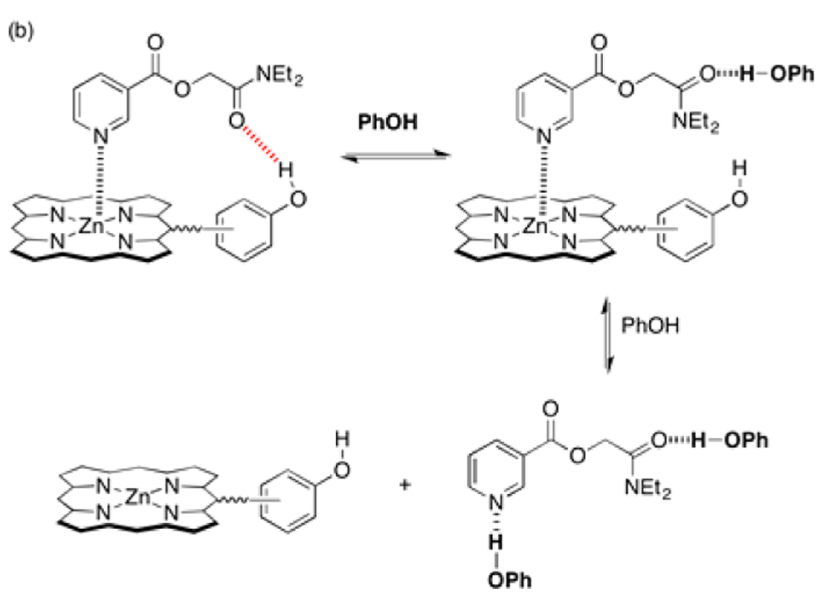

Figure 6. (a) Intermolecular phenol-amide H-bond formed between $p$-cresol and $N, N$-diethylacetamide used to estimate $K_{\text {ref. }}{ }^{24}$ (b) S2 $(\mathrm{PhOH})$ competes for both the intramolecular phenol-amide H-bond in complex A and the zinc-nitrogen coordination bond.

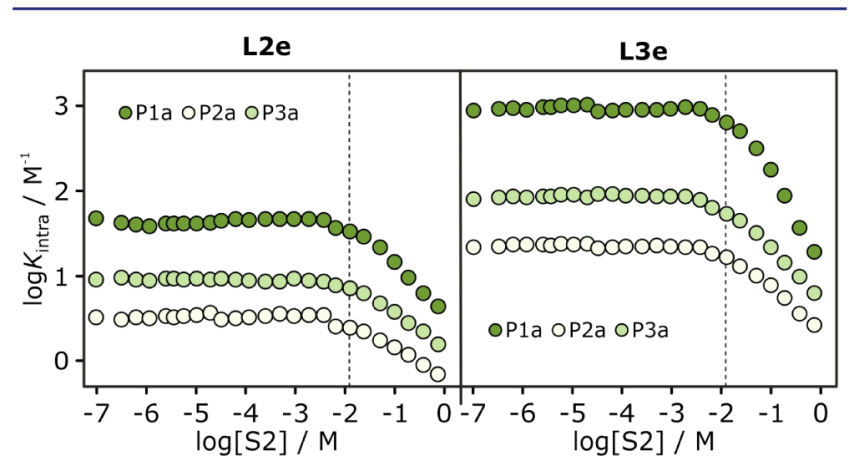

Figure 7. Values of $\log K_{\text {intra }}$ for formation of intramolecular amidephenol H-bonds plotted as a function of phenol concentration in toluene $(\log [\mathrm{S} 2])$.

concentrations less than $10 \mathrm{mM}$, but above this concentration, $\log K_{\text {intra }}$ decreases as the concentration of phenol increases. The slopes of the $\log K_{\text {intra }}$ versus $\log [\mathrm{S} 2]$ plots in this region are shown in Table 1 . The slopes for the $\mathbf{L} 3 \mathbf{e}$ complexes are

Table 1. Effective Molarities (EM) and Slopes $\left(m_{\text {exp }}\right)$ of $\log K_{\text {intra }}$ versus $\log [$ S2 $]$ Plots $^{24}$

\begin{tabular}{lccccc} 
& \multicolumn{2}{c}{ L2e } & & \multicolumn{2}{c}{ L3e } \\
\cline { 2 - 3 } \cline { 5 - 6 } & EM $(\mathrm{mM})$ & $m_{\exp }$ & & $\mathrm{EM}(\mathrm{mM})$ & $m_{\exp }$ \\
P1a & 140 & -0.6 & & 130 & -1.0 \\
P2a & 15 & -0.3 & & 14 & -0.5 \\
P3a & 36 & -0.4 & & 27 & -0.6 \\
P4a & & 0 & & 0 \\
\hline
\end{tabular}

approximately double the values found for the L2e complexes, as might be expected for systems that make twice as many $\mathrm{H}$ bonds. In the L2e complexes, the polar solvent competes with one intramolecular $\mathrm{H}$-bond, and in the L3e complexes S2 competes with two intramolecular $\mathrm{H}$-bonds. However, the slopes are all substantially less negative than might be expected based on the number of $\mathrm{H}$-bonds present in the complexes.
Within the L2e complexes the slopes vary by a factor of 2 between different porphyrins. Similarly the slopes for the L3e complexes span a range between -0.5 and -1.0 . The slope of the $\log K_{\text {intra }}$ versus $\log [\mathrm{S} 2]$ plot clearly does not provide the $\mathrm{H}$ bond inventory suggested in the Introduction.

Phenol can self-associate at high concentrations, and although the self-association constant in aromatic solvents is low, ${ }^{40}$ it is possible that aggregation could affect the results shown in Figure 7 and Table 1. In order to check the behavior of phenol at high concentrations in mixtures with toluene, the intermolecular $\mathrm{H}$-bond formed between 4-phenylazophenol and $\mathrm{N}, \mathrm{N}$-dihexylacetamide was studied in the same solvent mixtures. The results are shown in Figure 8. The behavior

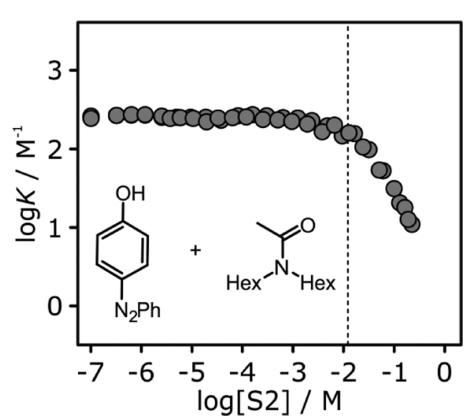

Figure 8. Values of $\log K$ for formation of a complex between 4phenylazophenol and $N, N$-dihexylacetamide as a function of phenol concentration in toluene $(\log [\mathrm{S} 2])$. The gradient of the linear part of the curve is -1 .

illustrated in Figure 1 is observed. When [S2] $<10 \mathrm{mM}, \log K$ is constant, and when $[\mathrm{S} 2]>10 \mathrm{mM}, \log K$ decreases in proportion to $\log [\mathrm{S} 2]$ with a slope of -1 . Since phenol is a good $\mathrm{H}$-bond donor and a poor $\mathrm{H}$-bond acceptor, the competition for formation of the intermolecular H-bond is dominated by interactions of $\mathrm{S} 2$ with the amide $\mathrm{H}$-bond acceptor. In other words, $K_{\mathrm{D}}$ in eq 1 is small, and $K_{\mathrm{A}}$ is large, which leads to the expected slope of -1 in the limit of large values of [S2] (eq 5).

$$
K \approx \frac{K_{\mathrm{S} 1}}{\left(1+K_{\mathrm{A}}[\mathrm{S} 2]\right)}
$$

where $K_{\mathrm{S} 1}=260 \mathrm{M}^{-1}$ and $K_{\mathrm{A}}=K_{\text {ref }}=86 \mathrm{M}^{-1}$ in toluene.

It should be possible to use eqs $1-5$ in the same way to rationalize the relationship between $\log K_{\text {intra }}$ and $\log [\mathrm{S} 2]$ observed for the intramolecular H-bonds in the porphyrinligand complexes. Neglecting the $K_{\mathrm{D}}$ term in eq 1 gives eq 5 , which successfully describes the data shown in Figure 8 for the intermolecular H-bond. Combining eqs 4 and 5 gives eq 6 for the L2e complexes that can make one intramolecular H-bond. Equation 7 is the equivalent relationship for the L3e complex that can make two intramolecular H-bonds.

$$
\begin{aligned}
& K_{\text {intra }}(\mathbf{L 2 e})=4 \frac{K_{\mathrm{S} 1} \mathrm{EM}}{1+K_{\mathrm{A}}[\mathrm{S} 2]} \\
& K_{\text {intra }}(\mathbf{L} 3 \mathbf{e})=8 \frac{K_{\mathrm{S} 1} \mathrm{EM}}{1+K_{\mathrm{A}}[\mathrm{S} 2]}+12\left(\frac{K_{\mathrm{S} 1} \mathrm{EM}}{1+K_{\mathrm{A}}[\mathrm{S} 2]}\right)^{2}
\end{aligned}
$$

Here, $K_{S 1}=K_{\mathrm{A}}=K_{\text {ref }}=86 \mathrm{M}^{-1}$ in toluene.

These equations show that when $K_{\mathrm{A}}[\mathrm{S} 2]<1$, the presence of the second polar solvent has no impact on the stability of complex A. When $K_{\mathrm{A}}[\mathrm{S} 2]>1$, the interaction of S2 with the 
amide groups of the free ligand competes for formation of the intramolecular $\mathrm{H}$-bond in complex A. For the systems described here, $K_{\mathrm{S} 1} \approx K_{\mathrm{A}}$, so the relative populations of the two zinc porphyrin-pyridine complexes shown in Figure $6 \mathrm{~b}$ are determined by the relationship between EM and [S2]. When [S2] > EM, the intramolecular H-bond will not be significantly populated because the amide groups of the ligand will preferentially interact with the solvent. When $[\mathrm{S} 2]<\mathrm{EM}$, the fully bound state of complex A with the intramolecular H-bond will dominate. However, even in systems where the intramolecular $\mathrm{H}$-bond is highly populated due to a high value of $\mathrm{EM}$, the presence of a polar solvent will still reduce the observed association constant for formation of complex A when $K_{\mathrm{A}}[\mathrm{S} 2]>1$, because the free ligand will be solvated by $\mathrm{S} 2$. Thus, the concentration at which S2 starts to affect the stability of a cooperatively assembled complex is determined by $K_{\mathrm{A}}$ and not by EM.

Equation 6 is similar to eq 5, which suggests that in the limit of large values of [S2], the slope of the $\log K_{\text {intra }}$ versus $\log [\mathrm{S} 2]$ plot for an intramolecular $\mathrm{H}$-bond should be -1 , as observed for the corresponding intermolecular $\mathrm{H}$-bond in Figure 8. However, the experimental slopes observed for the intramolecular $\mathrm{H}$-bonds formed by the one-armed ligand L2e are significantly less negative (Table 1 ). Moreover, eq 6 suggests that the limiting slope should be independent of the value of EM, which is not the case. For the two-armed ligand L3e, the relationship between the $\log K_{\text {intra }}$ and $\log [\mathrm{S} 2]$ is more complicated (eq 7). In the limit of large values of [S2], the slope is expected to be -2 when EM $>$ [S2] and -1 when EM $<$ [S2]. The experimental results in Table 1 are substantially less negative than these limiting values.

Equations 6 and 7 can be solved to determine values of EM as a function of [S2], and the results are shown in Figure 9. In

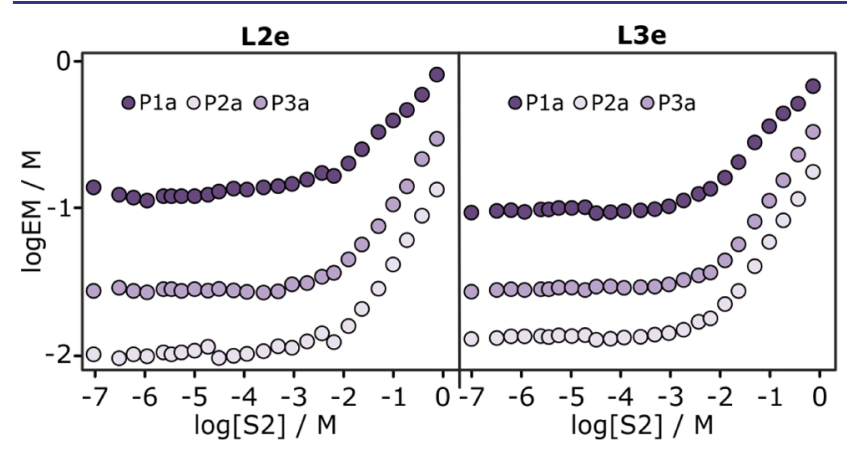

Figure 9. Relationship between EM for formation of intramolecular amide-phenol H-bonds as a function of phenol concentration in toluene $(\log [\mathrm{S} 2])$.

all cases, EM is not a constant but increases significantly with [S2] when the concentration of phenol exceeds $10 \mathrm{mM}$. The values of EM obtained for the one-armed and two-armed ligands are practically identical and vary in the same way with [S2]. Over the concentration range studied, EM increases by an order of magnitude, and the behavior is similar for all six of the porphyin-ligand complexes, which suggests that neither the overall supramolecular architecture nor the geometric complementarity play a significant role in determining the solvent dependence of EM.

Closer examination of the experimental data displayed in Figure 5 reveals that complex $\mathrm{C}$ is less stable than complexes $\mathrm{B}$ and D at high concentrations of S2 in all eight systems. Figure
10 shows the difference in the free energy of complexation of complexes $\mathrm{C}$ and $\mathrm{D}$ as a function of $[\mathrm{S} 2]\left(\Delta G_{\mathrm{C}}{ }^{\circ}-\Delta G_{\mathrm{D}}{ }^{\circ}\right)$. At

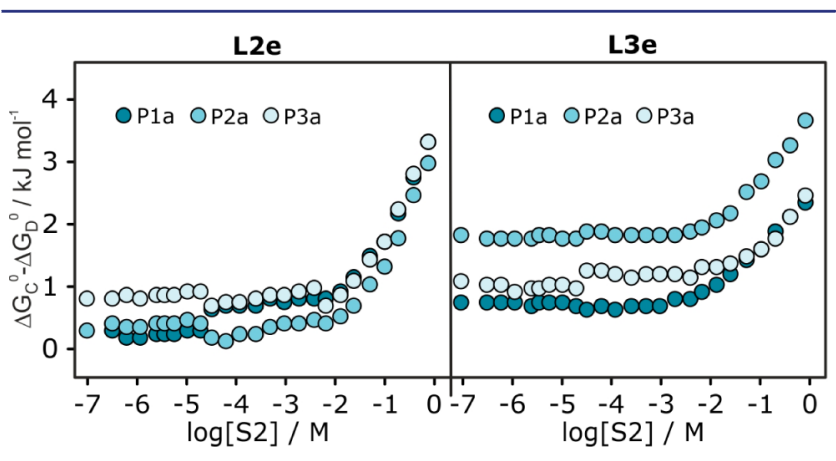

Figure 10. Free energy difference between complexes $\mathrm{C}$ and $\mathrm{D}$ of the DMC $\left(\Delta G_{C}{ }^{\circ}-\Delta G_{D}{ }^{\circ}\right)$ as a function of phenol concentration in toluene $(\log [\mathrm{S} 2])$.

low concentrations of S2, the differences are small, but when [S2] > $10 \mathrm{mM}$, significant concentration-dependent differences are observed. The positive value of $\Delta G_{C}{ }^{\circ}-\Delta G_{D}{ }^{\circ}$ indicates that there is a destabilization of complex $\mathrm{C}$ associated with the presence of the amide groups in the Le ligands. The shape of the plots in Figure 10 is similar to that of the plots in Figure 7, which suggests that the destabilization measured by the changes in $\Delta G_{\mathrm{C}}{ }^{\circ}-\Delta G_{\mathrm{D}}{ }^{\circ}$ are due to phenol binding to the amide groups of the ligands $\left(K_{\mathrm{A}}=86 \mathrm{M}^{-1}\right)$. There are no H-bonding interactions in complexes $\mathrm{C}$ and $\mathrm{D}$, so the differences must be related to steric effects associated with phenol binding to the amide groups. The steric effects are similar for all of the porphyrins indicating that it is interactions with the porphyrin core that are responsible rather than clashes with the substituents on the $m$-phenyl substituents.

Similar steric effects must be present when the amide ligands (Le) bind to the phenol-substituted porphyrins (Pa), and these interactions could explain the solvent-dependence of EM illustrated in Figure 9. The consequences of steric interactions with S2 are illustrated in Figure 11. When the amide acceptors

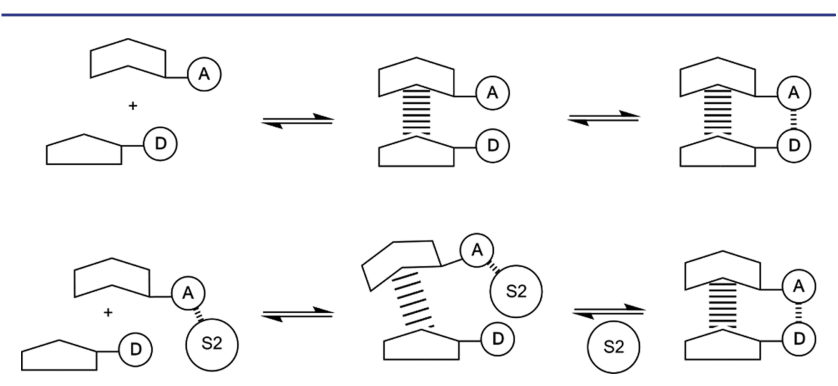

Figure 11. Schematic representation of steric effects associated with solvation by a polar solvent (S2) on intramolecular interactions between $\mathrm{A}$ and $\mathrm{D}$. When $\mathrm{A}$ is solvated by S2, the increased steric demand destabilizes the partially bound state, and this strain is relieved on formation of the A.D interaction in the fully bound state.

on the ligands (A) are solvated by phenol (S2), steric clashes between $\mathrm{S} 2$ and the porphyrin destabilize the partially bound state in which the zinc-nitrogen coordination bond is formed but the A.D H-bond is not. Formation of the A.D H-bond removes S2 and the steric clashes, leading to a higher equilibrium constant for the intramolecular process. The result is that, as the concentration of S2 increases and the ligands are increasingly solvated by $\mathrm{S} 2$, the intermediate partially bound 
state becomes less stable (cf. complex C), and EM for formation of the intramolecular interaction in complex A increases. These results are consistent with a previous observation that EMs measured for these systems in tetrachloroethane are somewhat larger than the values in other solvents. $^{30}$

\section{CONCLUSIONS}

The effects of preferential solvation on chelate cooperativity have been quantified using a family of zinc porphyrin-pyridine ligand complexes in mixtures of toluene and phenol. Chemical double mutant cycles have been used to measure the effective molarities for the formation of intramolecular phenol-amide $\mathrm{H}$-bonds in these systems. In all cases, EM increases dramatically with increasing concentrations of the more polar solvent, phenol. The result is that the effects of competitive interactions with polar solvents that reduce binding affinity are attenuated to a significant extent by a corresponding increase in EM in multivalent complexes.

The origin of the effect can be identified as strong solvation of the amide H-bond acceptor groups by the polar solvent, which increases the effective steric bulk of the ligands. These steric interactions have a destabilizing effect on the zinc porphyrin-pyridine complexes, but if an intramolecular $\mathrm{H}$ bond is made, the amide group must be desolvated, removing the adverse steric interactions. The result is an increase in EM by an order of magnitude compared with the corresponding intramolecular interactions in toluene.

The behavior observed in these systems is expected to be general: polar solvents will enhance cooperativity between multiple interaction sites that are in close proximity. In polar solvents, polar functional groups are strongly solvated. If multiple polar groups are in close proximity on the surface of a molecule, then formation of an intermolecular interaction at any one site will lead to steric interactions with the strongly bound solvation shell at neighboring sites. These adverse steric interactions will be removed, if all of the functional groups are desolvated to form cooperative intermolecular interactions. Thus, enhanced cooperativity is expected for strongly solvated systems.

\section{ASSOCIATED CONTENT}

\section{S Supporting Information}

The Supporting Information is available free of charge on the ACS Publications website at DOI: 10.1021/jacs.7b01765.

Detailed experimental procedures and tables (PDF)

\section{AUTHOR INFORMATION}

\section{Corresponding Author}

*herchelsmith.orgchem@ch.cam.ac.uk

\section{ORCID}

Stefan Henkel: 0000-0003-3362-8825

Maria Cristina Misuraca: 0000-0001-7024-4535

Christopher A. Hunter: 0000-0002-5182-1859

Notes

The authors declare no competing financial interest.

\section{ACKNOWLEDGMENTS}

We acknowledge financial support from the Engineering and Physical Sciences Research Council (EP/K025627/2). S.H. thanks the German Research Foundation (DFG) for a Postdoctoral Research Fellowship.

\section{REFERENCES}

(1) Persch, E.; Dumele, O.; Diederich, F. Angew. Chem., Int. Ed. 2015, 54 (11), 3290-3327.

(2) Ariga, K.; Ito, H.; Hill, J. P.; Tsukube, H. Chem. Soc. Rev. 2012, 41

(17), 5800-5835.

(3) Kay, E. R.; Leigh, D. A.; Zerbetto, F. Angew. Chem., Int. Ed. 2007, $46(1-2), 72-191$.

(4) Fasting, C.; Schalley, C. A.; Weber, M.; Seitz, O.; Hecht, S.; Koksch, B.; Dernedde, J.; Graf, C.; Knapp, E. W.; Haag, R. Angew. Chem., Int. Ed. 2012, 51 (42), 10472-10498.

(5) Badjic, J. D.; Nelson, A.; Cantrill, S. J.; Turnbull, W. B.; Stoddart, J. F. Acc. Chem. Res. 2005, 38 (9), 723-732.

(6) Philp, D.; Stoddart, J. F. Angew. Chem., Int. Ed. Engl. 1996, 35

(11), 1154-1196.

(7) Mulder, A.; Huskens, J.; Reinhoudt, D. N. Org. Biomol. Chem. 2004, 2 (23), 3409-3424.

(8) Mammen, M.; Choi, S. K.; Whitesides, G. M. Angew. Chem., Int. Ed. 1998, 37 (20), 2755-2794.

(9) O’Sullivan, M. C.; Sprafke, J. K.; Kondratuk, D. V.; Rinfray, C.; Claridge, T. D. W.; Saywell, A.; Blunt, M. O.; O’Shea, J. N.; Beton, P. H.; Malfois, M.; Anderson, H. L. Nature 2011, 469 (7328), 72-75.

(10) Gargano, J. M.; Ngo, T.; Kim, J. Y.; Acheson, D. W. K.; Lees, W. J. J. Am. Chem. Soc. 2001, 123 (51), 12909-12910.

(11) Dam, T. K.; Roy, R.; Das, S. K.; Oscarson, S.; Brewer, C. F. J. Biol. Chem. 2000, 275 (19), 14223-14230.

(12) Bundle, D. R.; Kitov, P. I.; Sadowska, J. M.; Mulvey, G.; Armstrong, G. D.; Ling, H.; Pannu, N. S.; Read, R. J. Nature 2000, 403 (6770), 669-672.

(13) Anderson, H. L. Inorg. Chem. 1994, 33 (5), 972-981.

(14) De Greef, T. F. A.; Smulders, M. M. J.; Wolffs, M.; Schenning, A.; Sijbesma, R. P.; Meijer, E. W. Chem. Rev. 2009, 109 (11), 56875754.

(15) Mahadevi, A. S.; Sastry, G. N. Chem. Rev. 2016, 116 (5), 27752825.

(16) Motloch, P.; Hunter, C. A., Thermodynamic Effective Molarities for Supramolecular Complexes. In Advances in Physical Organic Chemistry; Williams, I. H., Williams, N. H., Eds.; Academic Press, 2016; Vol. 50, pp 77-118.

(17) Hunter, C. A.; Anderson, H. L. Angew. Chem., Int. Ed. 2009, 48 (41), 7488-7499.

(18) Hogben, H. J.; Sprafke, J. K.; Hoffmann, M.; Pawlicki, M.; Anderson, H. L. J. Am. Chem. Soc. 2011, 133 (51), 20962-20969.

(19) Ercolani, G. J. Phys. Chem. B 2003, 107 (21), 5052-5057.

(20) Ercolani, G.; Schiaffino, L. Angew. Chem., Int. Ed. 2011, 50 (8), $1762-1768$.

(21) Hunter, C. A.; Ihekwaba, N.; Misuraca, M. C.; Segarra-Maset, M. D.; Turega, S. M. Chem. Commun. 2009, 26, 3964-3966.

(22) Sun, H. M.; Hunter, C. A.; Llamas, E. M. Chem. Sci. 2015, 6 (2), $1444-1453$.

(23) Sun, H. M.; Guo, K.; Gan, H. F.; Li, X.; Hunter, C. A. Org. Biomol. Chem. 2015, 13 (29), 8053-8066.

(24) Adams, H.; Chekmeneva, E.; Hunter, C. A.; Misuraca, M. C.; Navarro, C.; Turega, S. M. J. Am. Chem. Soc. 2013, 135 (5), 18531863.

(25) Sun, H. M.; Navarro, C.; Hunter, C. A. Org. Biomol. Chem. 2015, 13 (17), 4981-4992.

(26) Misuraca, M. C.; Grecu, T.; Freixa, Z.; Garavini, V.; Hunter, C. A.; van Leeuwen, P.; Segarra-Maset, M. D.; Turega, S. M. J. Org. Chem. 2011, 76 (8), 2723-2732.

(27) Hunter, C. A.; Misuraca, M. C.; Turega, S. M. J. Am. Chem. Soc. 2011, 133 (3), 582-594.

(28) Hunter, C. A.; Misuraca, M. C.; Turega, S. M. J. Am. Chem. Soc. 2011, 133 (50), 20416-20425.

(29) Hunter, C. A.; Misuraca, M. C.; Turega, S. M. Chem. Sci. 2012, 3 (2), 589-601. 
(30) Chekmeneva, E.; Hunter, C. A.; Misuraca, M. C.; Turega, S. M. Org. Biomol. Chem. 2012, 10 (30), 6022-6031.

(31) Hunter, C. A.; Misuraca, M. C.; Turega, S. M. Chem. Sci. 2012, 3 (8), 2462-2469.

(32) Sun, H.; Hunter, C. A.; Navarro, C.; Turega, S. J. Am. Chem. Soc. 2013, 135 (35), 13129-13141.

(33) Jinks, M. A.; Sun, H. M.; Hunter, C. A. Org. Biomol. Chem. 2014, 12 (9), 1440-1447.

(34) Cabot, R; Hunter, C. A. Chem. Soc. Rev. 2012, 41 (9), 34853492.

(35) Amenta, V.; Cook, J. L.; Hunter, C. A.; Low, C. M. R.; Vinter, J. G. Org. Biomol. Chem. 2011, 9 (21), 7571-7578.

(36) Amenta, V.; Cook, J. L.; Hunter, C. A.; Low, C. M. R.; Vinter, J. G. J. Phys. Chem. B 2012, 116 (49), 14433-14440.

(37) Amenta, V.; Cook, J. L.; Hunter, C. A.; Low, C. M. R.; Sun, H.; Vinter, J. G. J. Am. Chem. Soc. 2013, 135 (32), 12091-12100.

(38) Cockroft, S. L.; Hunter, C. A. Chem. Soc. Rev. 2007, 36 (2), 172-188.

(39) Camara-Campos, A.; Musumeci, D.; Hunter, C. A.; Turega, S. J. Am. Chem. Soc. 2009, 131 (51), 18518-18524.

(40) Magee, M. D.; Walker, S. Can. J. Chem. 1971, 49 (7), 11061114. 